\title{
PD11 - Omalizumab in children: experience in the Immunoallergology Department
}

\author{
Rita Aguiar ${ }^{*}$, Pedro Silva, Fátima Cabral Duarte, Ana Mendes, Ana Célia Costa, Manuel Barbosa Pereira \\ From 3rd Pediatric Allergy and Asthma Meeting (PAAM) \\ Athens, Greece. 17-19 October 2013
}

\section{Introduction}

Omalizumab is a monoclonal antibody approved for the treatment of severe allergic asthma for patineis above 6 years old in Europe, but its use has been described successfully in other IgE-mediated diseases.

\section{Objective}

Evaluation of clinical response and safety of treatment with omalizumab in a pediatric population.

\section{Methods}

Retrospective analysis of clinical data of pts aged $<18$ years at the time of initiation of treatment with omalizumab in our department from December 2009 to July 2013. We evaluate adverse reactions, clinical outcome, reduction or discontinuation of therapy according to allergic disease.

\section{Results}

Ten pts were proposed to omalizumab: $8 \mathrm{M}, 2 \mathrm{~F}$ with a mean age of 12.9 years \pm 3.9 [7-17]. All had bronchial asthma: 3severe allergic asthma, 5severe atopic eczema (AE), 1severe ocular allergy (OA) with risk for injury to the cornea by conjunctival edema and 1 cow's milk protein allergy (CMA) with anaphylaxis during the protocol for oral tolerance induction (OTI).

The mean duration of therapy with omalizumab was $12.3 \pm 13.35$ months. The median serum total IgE 1 before treatment was $991.5 \mathrm{kU} / \mathrm{L}$.

The average values of Asthma Control Test (ACT) before and after omalizumab were 17 [15-24]. The mean Scoring Atopic Dermatitis (SCORAD) before and after omalizumab was 2 [34-90] and 24 [13-35].

The pts with EA, 1 was treated with cyclosporine, 1 azathioprine, 2 with systemic corticosteroids (SC). After an average of 2 months of treatment there was a reduction

$$
\text { Department of Immunoallergology, Hospital de Santa Maria, CHLN, Lisbon, }
$$$$
\text { Portugal }
$$ 